\title{
Biopsy of Different Oral Soft Tissues Lesions by KTP and Diode Laser: Histological Evaluation
}

\author{
Umberto Romeo, ${ }^{1}$ Claudia Russo, ${ }^{1}$ Gaspare Palaia, ${ }^{1}$ Rossella Lo Giudice, ${ }^{1}$ \\ Alessandro Del Vecchio, ${ }^{1}$ Paolo Visca, ${ }^{2}$ Guido Migliau, ${ }^{1}$ and Alberto De Biase ${ }^{1}$ \\ ${ }^{1}$ Department of Oral and Maxillofacial Sciences, "Sapienza” University of Rome, Via Caserta 6, 00161 Rome, Italy \\ ${ }^{2}$ Department of Cytology and Cellular Diagnostics, Regina Elena Institute, Via Elio Chianesi 53, 00144 Rome, Italy
}

Correspondence should be addressed to Gaspare Palaia; gaspare.palaia@uniroma1.it

Received 29 July 2014; Accepted 9 September 2014; Published 27 October 2014

Academic Editor: Samir Nammour

Copyright (C) 2014 Umberto Romeo et al. This is an open access article distributed under the Creative Commons Attribution License, which permits unrestricted use, distribution, and reproduction in any medium, provided the original work is properly cited.

\begin{abstract}
Introduction. Oral biopsy aims to obtain clear and safe diagnosis; it can be performed by scalpel or laser. The controversy in this latter application is the thermal alteration due to tissue heating. The aim of this study is the histological evaluation of margins of "in vivo" biopsies collected by diode and KTP lasers. Material and Methods. 17 oral benign lesions biopsies were made by diode $808 \mathrm{~nm}$ (SOL, DenMatItalia, Italy) and KTP $532 \mathrm{~nm}$ (SmartLite, DEKA, Italy). Samples were observed at OM LEICA DM 2000; margin alterations were evaluated through Leica Application Suite 3.4. Results. Epithelial and connective damages were assessed for each pathology with an average of $0.245 \mathrm{~mm}$ and a standard deviation of $\pm 0.162 \mathrm{~mm}$ in mucoceles, $0.382 \mathrm{~mm} \pm 0.149 \mathrm{~mm}$ in fibromas, $0.336 \mathrm{~mm} \pm$ $0.106 \mathrm{~mm}$ in hyperkeratosis, $0.473 \mathrm{~mm} \pm 0.105 \mathrm{~mm}$ in squamous hyperplasia, $0.182 \mathrm{~mm}$ in giant cell granuloma, and $0.149 \mathrm{~mm}$ in melanotic macula. Discussion. The histologic aspect of lesions influenced the response to laser, whereas the greater inflammation and cellularity were linked with the higher thermal signs. Many artifacts were also associated to histologic procedures. Conclusion. Both tested lasers permitted sure histologic diagnosis. However, it is suggested to enlarge biopsies of about $0.5 \mathrm{~mm}$, to avoid thermal alterations, especially in inflammatory lesions like oral lichen planus.
\end{abstract}

\section{Introduction}

A biopsy is a diagnostic procedure which consists in taking a tissue fragment to subject it to a histological examination and, therefore, to obtain a diagnosis of certainty that can or cannot confirm the suspicion clinical diagnostic [1].

Biopsies can be classified according to the used material, the clinician timing, the lesion site, and the used technique that can be distinguished in incisional and excisional biopsies. The incisional biopsy involves the removal of a representative portion of the lesion and a portion of healthy tissue adjacent to it [2-4]; while the excisional biopsy consists in the removal of the whole lesion allowing, at the same time, carrying out both a diagnostic and therapeutic procedure $[4,5]$.

The biopsy is generally indicated for the following:

(i) recognizing neoplastic, preneoplastic, and other soft tissue diseases; (ii) identifying the origin of ulcers that do not heal within two weeks;

(iii) defining the nature of lesions that do not regress after therapy;

(iv) removing lesions of the right dimensions and verifying their nature.

Nowadays it is possible to perform oral biopsies using two different tools, the scalpel and the laser.

The scalpel allows obtaining a tissue fragment characterized by the presence of well-defined peri-incisional margins with no structural alterations. However, this surgery always requires anesthesia and sutures, and the operative field is not bloodless.

The laser devices most commonly used in oral soft tissues surgery are the diode $(600-980 \mathrm{~nm})$, the potassium titanyl phosphate (KTP, $532 \mathrm{~nm}$ ), the carbon dioxide laser $\left(\mathrm{CO}_{2}, 10600 \mathrm{~nm}\right)$, the neodymium-doped yttrium aluminum 
garnet (Nd:YAG, $1064 \mathrm{~nm}$ ), and the erbium-doped yttrium aluminum garnet (Er:YAG $2940 \mathrm{~nm}$ ).

Lasers, used for biopsies execution, have several advantages than the scalpel. In fact, they consent to obtain a good hemostasis, bloodless field and a faster healing, above all during the initial phases [6].

However, due to the thermal effects of the laser, incisional margins of tissue samples can be altered, creating doubts about the effectiveness of this method in the diagnosis of systemic disease [7]. If the use of this tool has many advantages over the cold blade, the risk of jeopardizing the outcome of histological analysis, due to laser thermal effects on peri-incisional area, still raises doubts. Actual scientific literature does not reveal in vivo studies concerning the evaluation of peri-incisional biopsy taken with the laser. Pathological tissues "in vivo," compared to those "ex vivo," are characterized by a higher concentration of liquid, lower cell cohesion, and normal or pathological amounts of blood (e.g., in inflammatory or autoimmune diseases).

This consideration could lead to an improvement in cutting ability of the laser that could permit the parameters applied to be reduced with less damage to cut margins but on the other hand to higher local heat buildup with larger thermal artefacts.

The aim of this "in vivo" study is to analyze the tissue fragments removed by laser surgery, to assess the epithelial and connective tissue damage caused by its thermal effects.

\section{Materials and Methods}

Seventeen patients $(8 \mathrm{~F} / 9 \mathrm{M})$, affected by oral benign pathologies, have been subjected to oral excisional biopsy. In some cases, lesions have been treated using an incisional biopsy because of their site or their size. All tissue samples have been removed by the same operator in order to execute a proper biopsy thanks to his experience and knowledge in laser tools and biological tissue characteristics.

Biopsies have been performed using two different wavelengths with the following parameters: diode laser $808 \mathrm{~nm}$ (SOL, DenMatItalia, Italy), power: $2 \mathrm{~W}$ in CW, fluence: $2400 \mathrm{~J} / \mathrm{cm}^{2}$, fiber spot: $320 \mu \mathrm{m}$; KTP laser $532 \mathrm{~nm}$ (SmartLite, DEKA, Italy), power: $1.5 \mathrm{~W}$ in PW, fluence: $212 \mathrm{~J} / \mathrm{cm}^{2}$, fiber spot: $300 \mu \mathrm{m}$. Parameters have been selected considering the right execution of the surgical intervention and the patient compliance never exceeding 5 minutes.

Local anesthesia with $1.8 \mathrm{~mL}$ of mepivacaine solution (Mepivacaina Pierrel, $30 \mathrm{mg} / \mathrm{mL}$, injection solution $1.8 \mathrm{~mL}$, Pierrel Spa, Milan, Italy) without vasoconstrictor was performed around the area of the lesion before the beginning of each surgical intervention, injecting the solution at a distance of $0,5 \mathrm{~cm}$ from lesions margins. The excised lesions size was between 0,5 and $1 \mathrm{~cm}$ of diameter. Two mucoceles were taken out by diode laser and one by KTP laser; 5 fibromas were excised by diode; 3 hyperkeratosis lesions were removed by diode and 1 by KTP; the 3 oral lichen planus, the melanotic macula, and the oral giant cell granuloma were removed by diode laser. After surgery, the samples were sent to the pathologist, for the histological evaluation and diagnosis, in a single-blind mode. No suture or medication was applied and the wound was left to heal by secondary intention. All biopsy samples were fixed in a $10 \%$ neutral-buffered formalin solution, embedded in paraffin, sectioned, and stained with hematoxylin-eosin for conventional histopathological evaluation.

Tissue fragments were again observed through the use of an optical microscope LEICA DM 2000, 5x and 10x magnification, and thanks to an appropriate software (Leica Application Suite version 3.4) quantitatively and qualitatively marginal alterations, due to the thermal action of lasers, have been evaluated. Quantitative evaluation carried out a measurement in millimeters and statistical analysis was carried out by calculating the arithmetic mean and standard deviation (a measure of the dispersion of data around the expected value) of the different values, while the involvement of epithelial and connective tissue in thermal alterations has been evaluated in the qualitative aspect. In every oral pathology, connective and epithelial damage have been evaluated in terms of charring and coarctation, since in many cases it was impossible to evaluate them separately.

\section{Results}

Follow-up at 7 and 21 days showed a complete recovery of the wound, without any complications or pain.

The presence of peripheral alterations has not influenced histological analysis: for all samples, it was possible to obtain a certainty diagnosis. Histological examination showed three mucoceles, five fibromas, four hyperkeratotic lesions, three oral lichen planus, one giant cells granuloma, and one melanotic macula.

Peripheral damage has been individually evaluated for each disease, considering that the morphological and structural characteristics of the various lesions could strongly influence the tissues response to the laser action.

Graphs have been realized to show the trend of the measures, and statistical analysis has been carried out calculating the mean and standard deviation of the different values. Moreover, in each histological group the same parameters have been evaluated.

The histological evaluation of peri-incisional margins in which the microscopic analysis was compatible with the diagnosis of mucocele showed a damage average of $0.245 \mathrm{~mm}$ with a standard deviation of $\pm 0.162 \mathrm{~mm}$ (Figures 1 and 2; Table 1). Only in one case the epithelium was not visible because the damage was exclusively assessed to the connective tissue.

In the clinical cases in which the histological evaluation leads to diagnosis of fibromas (Figures 3 and 4; Table 2) the damage average was of $0.382 \mathrm{~mm}$ and the standard deviation was of $0.149 \mathrm{~mm}$. Also in one of these cases, it was not possible to consider the epithelium of a sample because the damage has been measured only in the connective layer.

Histological evaluation of the peri-incisional margins of hyperkeratotic lesions (Figures 5 and 6; Table 3), compatible with the diagnosis of squamous hyperplasia, showed a damage average of $0.336 \mathrm{~mm}$ with a standard deviation of $\pm 0.106 \mathrm{~mm}$.

Furthermore, the histological evaluation of the periincisional margins of clinical cases whose diagnosis was oral 
TABLE 1: Mucocele biopsies.

\begin{tabular}{lcccc}
\hline Patient & Sex & Lesion site & Laser & Damage (in mm) \\
\hline D.C. & F & Inferior lip & Diode 808 & 0,442 \\
N.F. & F & Upper left lip & KTP & 0,213 \\
S.S. & M & Inferior lip & Diode 808 & 0,102 \\
\hline
\end{tabular}

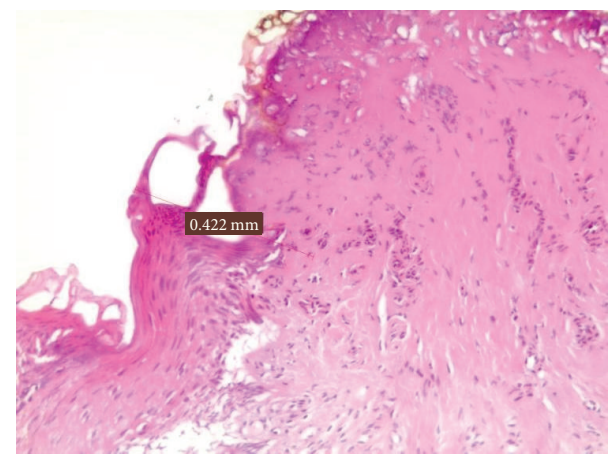

FIgURE 1: Damage measurement in a mucocele.

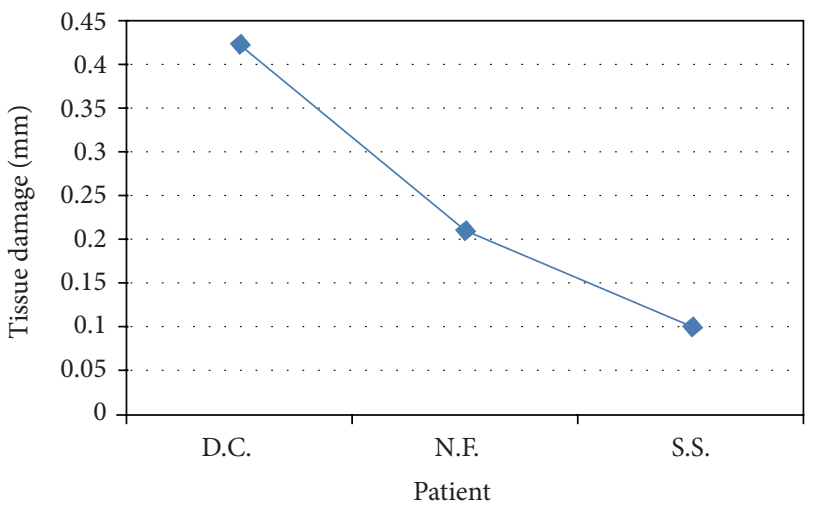

Figure 2: Peri-incisional marginal damage in mucocele.

lichen planus demonstrated a damage average of $0.473 \mathrm{~mm}$ with a standard deviation of $\pm 0.105 \mathrm{~mm}$ (Figures 7 and 8; Table 4).

The damage average in the microscopic examination of the giant cell granuloma was $0.182 \mathrm{~mm}$ (Figure 9).

Finally, in the melanotic macula, removed by the diode laser $808 \mathrm{~nm}$, the damage average was equal to $0.149 \mathrm{~mm}$ (Figure 10).

\section{Discussion}

Several studies are present in the literature about [8-11] the use of laser in oral soft tissue biopsy, but only few of them focus on the damage caused by this device at periincisional margins of tissue fragments. Every type of laser can create thermal damage to the target tissues because of the photothermal effect. While lasers work, they heat tissues, causing a temperature increase, at the point of incidence, of more than 100 degrees. Surrounding tissues can be involved in the increase of temperature and so they are permanently
TABLE 2: Fibroma biopsies.

\begin{tabular}{lcccc}
\hline Patient & Sex & Lesion site & Laser & Damage (in mm) \\
\hline B.M.L. & F & Left cheek & Diode 808 & 0,411 \\
D.G. & M & Left cheek & Diode 808 & 0,357 \\
L.R. & F & Left tongue margin & Diode 808 & 0,267 \\
O.G. & F & Left cheek & Diode 808 & 0,623 \\
O.M. & M & Right cheek & Diode 808 & 0,252 \\
\hline
\end{tabular}

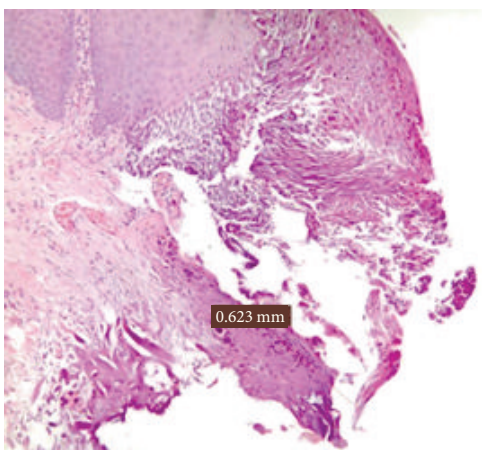

Figure 3: Damage measurement in a fibroma.

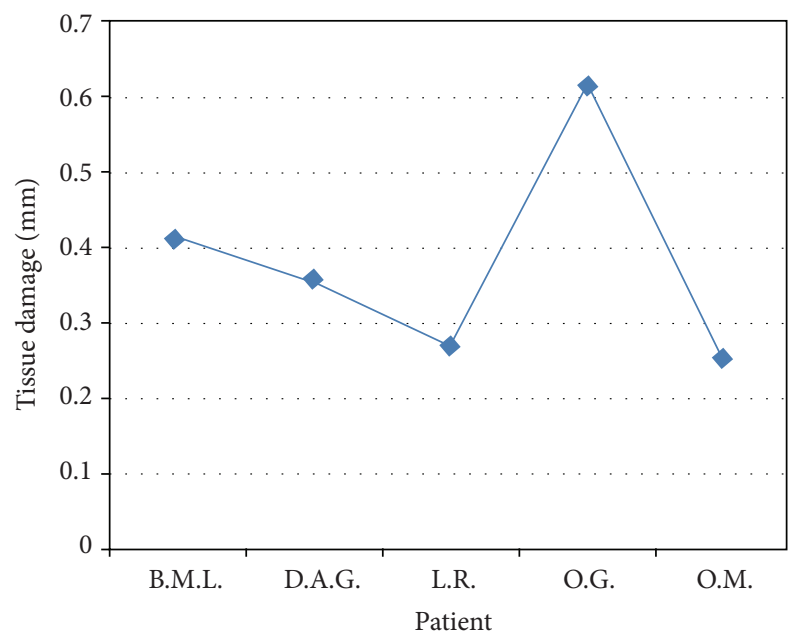

FIgURE 4: Representation of peri-incisional marginal damage in fibroma.

or reversibly damaged. Furthermore, the histologic exam is linked to the integrity of peri-incisional margins, and this is a basic requirement for a tool employed in biopsies.

A study carried out on rabbits by Rizoiu et al. [11] showed no differences in the histology among peri-incisional margins of samples excised by laser and by scalpel. In a Dalrymple and Russell's study [12], about the evaluation of peri-incisional margins of incisional and excisional biopsies performed by $\mathrm{CO}_{2}$ laser on cervix lesions, it appeared that marginal alterations were on average $0.3 \mathrm{~mm}$. However, due to thermal damage in $12 \%$ of cases the histological examination gave an uncertain outcome.

In a study carried out by Romeo et al. [13], the effects of Er:YAG, Nd:YAG, Er-Cr:YSGG, and two diode lasers (resp., 
TABle 3: Hyperkeratotic lesion biopsies.

\begin{tabular}{lcccc}
\hline Patient & Sex & Lesion site & Laser & Damage (in $\mathrm{mm}$ ) \\
\hline M.B. & M & Right tongue margin & Diode 808 & 0,319 \\
M.M. & M & Lower lip & Diode 808 & 0,446 \\
Z.R. & F & Lower left lip & KTP & 0,196 \\
N.C. & M & Right cheek & Diode 808 & 0,383 \\
\hline
\end{tabular}

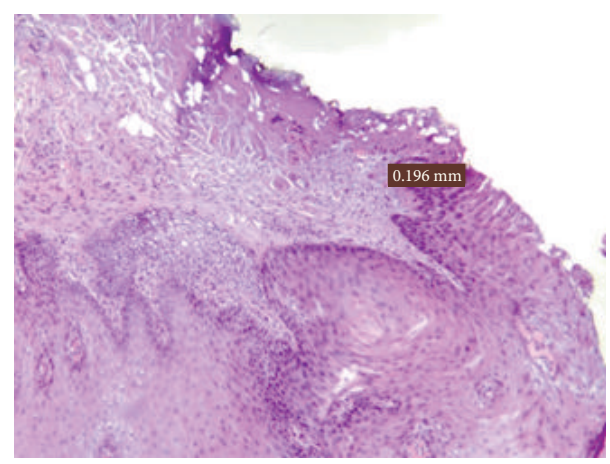

FIGURE 5: Damage measurement in a hyperkeratotic lesion.

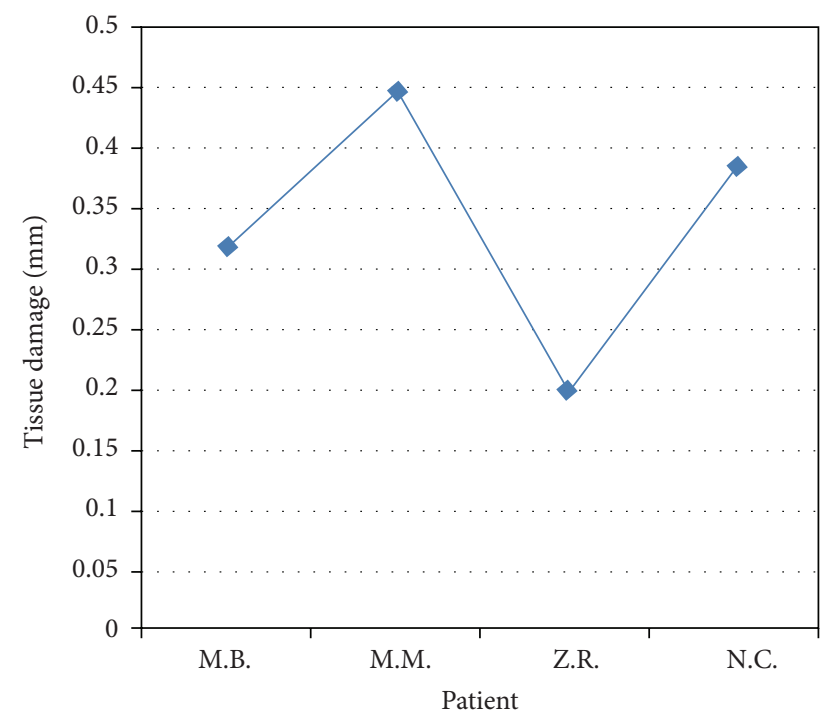

Figure 6: Peri-incisional marginal damage in hyperkeratotic lesion.

$808 \mathrm{~nm}$ and $980 \mathrm{~nm}$ ) have been evaluated on pig tongue. It resulted in the fact that each kind of laser device could be used to perform biopsy. Even if they caused slight alterations in the taken tissue margins, no one of them compromised the histological evaluation. In particular, the best results have been obtained with the $808 \mathrm{~nm}$ diode laser device in pulsed wave mode and with Er-Cr:YSGG laser at higher power, which created peripheral damage less than $1 \mathrm{~mm}$.

Another study carried out by Romeo et al. [14] about the histological evaluation of Er:YAG laser effect on oral soft tissues showed that, using this device with intermediate power $(80-100 \mathrm{~mJ})$, the thermal damage was always under the millimeter involving only the epithelium layer. So, authors
TABLE 4: Oral lichen planus biopsies.

\begin{tabular}{lcccc}
\hline Patient & Sex & Lesion site & Laser & Damage (in mm) \\
\hline D.G.C. & F & Right cheek & Diode 808 & 0,504 \\
L.L. & F & Right cheek & Diode 808 & 0,356 \\
M.M. & M & Right cheek & Diode 808 & 0,561 \\
\hline
\end{tabular}

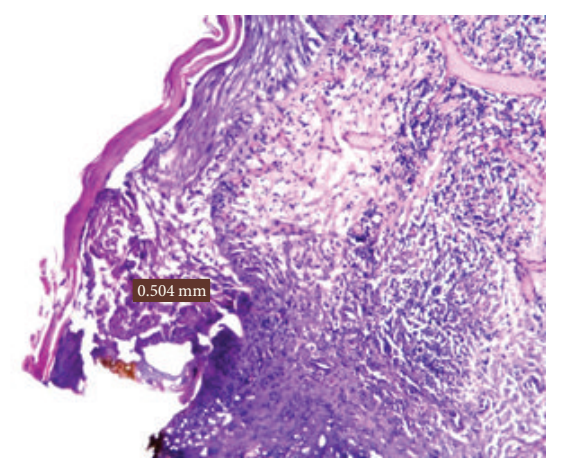

Figure 7: Damage measurement in an oral lichen planus.

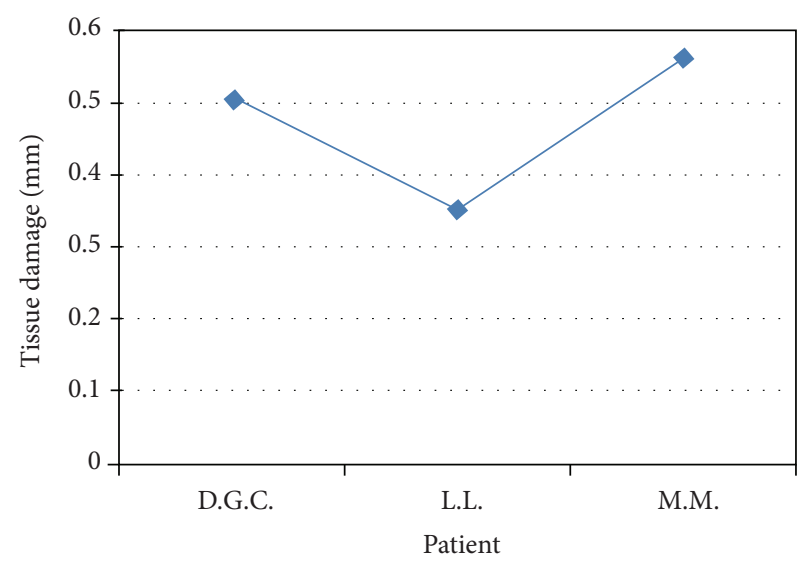

FIGURE 8: Representation of peri-incisional marginal damage in oral lichen planus.

concluded that thermal damages was negligible and the readability of the perioincisional margins was always possible.

Moreover, a study about the effects of the KTP [15] on oral soft tissue demonstrated that it allowed the execution of precise cut provoking a minimum cellular damage in the epithelium and in the chorion. The precision of the obtained margins make them similar to those ones obtained through the use of a scalpel. In addition to this, specimens of all tested groups were free from thermal artefacts above all when lowest fluence settings have been used.

Furthermore, in a study carried out by Merigo et al. [16] concerning the use of different wavelengths in laser-assisted surgery, it was shown that positive results have been obtained for the evaluation of laser-excised samples in terms of their readability and diagnostic reliability.

Vescovi et al. [17] performed a preliminary histological analysis of specimens from the human oral mucosa comparing Nd:YAG laser versus traditional scalpel. Epithelial 


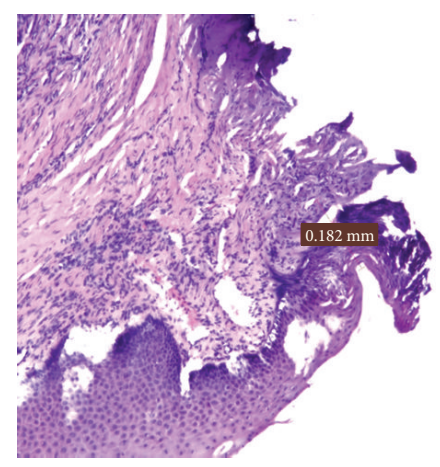

FIGURE 9: Damage measurement in a giant cells granuloma.

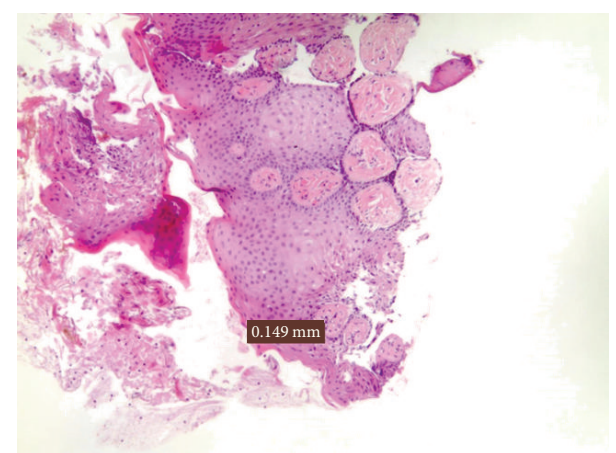

Figure 10: Damage measurement in a melanotic macula.

changes, connective tissue modifications, presence of vascular modifications, incision morphology, and the overall width of tissue modification were evaluated. Differences between specimens removed with two different parameters of Nd:YAG ( $3.5 \mathrm{~W}, 60 \mathrm{~Hz}$ and $5 \mathrm{~W}, 30 \mathrm{~Hz}$ ) laser were not significant with regard to stromal changes and vascular stasis. The quality of incision was better and the width of overall tissue injuries was less in the specimens obtained with higher frequency and lower power (group 1: Nd:YAG laser at $3.5 \mathrm{~W}$ and $60 \mathrm{~Hz}$ ).

In a retrospective study, Angiero et al. [18], 608 cases of soft tissue lesions localized in the oral cavity (cheek, gingiva, buccal mucosa, tongue, and lips) were examined. Specimens were excised with an $808 \mathrm{~nm}$ diode laser, output 1.6-2.7 W, in continuous-wave mode with fibers of $320 \mu \mathrm{m}$. The data for specimens larger than $3 \mathrm{~mm}$ excised with the diode laser were not significant in terms of stromal changes or vascular stasis, while epithelial and stromal changes were significantly more frequent in specimens with a mean size below $3 \mathrm{~mm}$. Authors suggest that the specimens taken have "in vivo" a diameter of at least $5 \mathrm{~mm}$ in order to have a reliable reading of the histological sample, but this recommendation is valid even for a scalpel biopsy.

According to several studies the possibility to evaluate "in vivo" the marginal alterations of samples excised by laser is not clear. For this reason, there was a necessity to begin an "in vivo" study concerning the histological exam of periincisional margins after laser biopsy.

This study showed that the biopsy of oral soft tissues, performed by diode or KTP laser, did not create any significant marginal alterations that could compromise the histological diagnosis. Moreover, it shows, as explained in the previous "ex vivo" study [13], that the laser device that causes less thermal damage is the KTP. In general, in fact, the laser tissue interaction is due to the operator-dependent factors (modality of use, application time, and choice of the cutting distance from the lesion margins) and the operatorindependent factors related to the wavelength and to the optical properties of the tissue.

The bioptic samples of this study showed that carbonization and coarctation were more limited in specific lesions (mucocele) than in others (oral lichen planus), demonstrating in this way how the increased cellularity and inflammation, typical of some lesions, can cause an increase of the periincisional damage.

Moreover, it is important to consider that many of the artifacts, found on the samples, were not due to the action of the laser but due to problems which occurred during the process of fixing, cutting, and staining of the tissue fragment.

Finally, the use of laser devices is not advisable to perform biopsies of suspicious lesions. In this case, the analysis of cellular infiltration in the adjacent tissues is fundamental and the thermal effects of the laser may affect the possibility to realize a proper analysis of the lesion margins and to establish the real cancer size $[8,19,20]$.

\section{Conclusions}

Laser devices, used by a skilled operator, allow obtaining histological tissue fragments with important advantages both for the operator and for the patient. In fact, thanks to the laser-haemoglobin interaction, the surgical field is bloodless, permitting having greater visibility and also performing surgery in patients affected by coagulation disorders. Furthermore, it is possible to reduce the amount of local anesthesia and to achieve a faster postoperative healing, especially in the early stages.

In this study, it was always possible to obtain a sure histological diagnosis for each sample.

So, laser devices, because of their excellent surgical properties, can be used successfully to perform oral soft tissues biopsies, but a clinical preliminary analysis of the lesion is fundamental, in order to predict whether the peri-incisional thermal damage will be more or less extended. However, the peri-lesional damage did not compromise the morphological and structural characteristics of the specimens.

\section{Conflict of Interests}

The authors declare that there is no conflict of interests regarding the publication of this paper.

\section{References}

[1] A. García-Peñín, "Biopsia en cirugía bucal," in Cirugía Bucal: patología y técnica, M. Donado, Ed., pp. 119-131, Masson, 1990.

[2] A. Mota-Ramírez, F. J. Silvestre, and J. M. Simó, "Oral biopsy in dental practice," Medicina Oral, Patología oral y Cirugía Bucal, vol. 12, no. 7, pp. E504-E510, 2007. 
[3] R. J. Oliver, P. Sloan, and M. N. Pemberton, "Oral biopsies: methods and applications," British Dental Journal, vol. 196, no. 6, pp. 329-333, 2004.

[4] R. J. Melrose, J. P. Handlers, S. Kerpel, D.-J. Summerlin, and C. J. Tomich, "The use of biopsy in dental practice: the position of the American Academy of Oral and Maxillofacial Pathology," General Dentistry, vol. 55, no. 5, pp. 457-461, 2007.

[5] C. Scully, Oral and Maxillofacial Medicine, the Basis of Diagnosis and Treatment, 3: Oral and Maxillofacial Medicine, Elsevier Health Sciences, 2013.

[6] R. J. Fonseca, S. B. Baker, and L. M. Wolford, Oral and Maxillofacial Surgery, vol. 6, W.B. Saunders, 2000.

[7] P. Sahni, "Lasers in oral pathology-a review," Medico-Legal Update, vol. 12, no. 1, pp. 65-67, 2012.

[8] M. M. Bornstein, C. Winzap-Kälin, D. L. Cochran, and D. Buser, "The $\mathrm{CO}_{2}$ laser for excisional biopsies of oral lesions: a case series study," The International Journal of Periodontics \& Restorative Dentistry, vol. 25, no. 3, pp. 221-229, 2005.

[9] H. M. Saleh and A. M. Saafan, "Excision biopsy of tongue lesions by diode laser," Photomedicine and Laser Surgery, vol. 25, no. 1, pp. 45-49, 2007.

[10] A. L. Pinheiro and J. W. Frame, "Surgical management of premalignant lesions of the oral cavity with the $\mathrm{CO}_{2}$ laser," Brazilian Dental Journal, vol. 7, no. 2, pp. 103-108, 1996.

[11] I. M. Rizoiu, L. R. Eversole, and A. I. Kimmel, "Effects of an erbium, chromium: yttrium, scandium, gallium, garnet laser on mucocutanous soft tissues," Oral Surgery, Oral Medicine, Oral Pathology, Oral Radiology, and Endodontics, vol. 82, no. 4, pp. 386-395, 1996.

[12] C. Dalrymple and P. Russell, "Thermal artefact after diathermy loop excision and laser excision cone biopsy," International Journal of Gynecological Cancer, vol. 9, no. 3, pp. 238-242, 1999.

[13] U. Romeo, A. Del Vecchio, F. Ripari et al., "Effects of different laser devices on oral soft tissues: in vitro experience," The Journal of Oral Laser Applications, vol. 7, no. 3, pp. 155-159, 2007.

[14] U. Romeo, F. Libotte, G. Palaia et al., "Histological in vitro evaluation of the effects of Er:YAG laser on oral soft tissues," Lasers in Medical Science, vol. 27, no. 4, pp. 749-753, 2012.

[15] U. Romeo, G. Palaia, A. Del Vecchio et al., "Effects of KTP laser on oral soft tissues. An in vitro study," Lasers in Medical Science, vol. 25, no. 4, pp. 539-543, 2010.

[16] E. Merigo, F. Clini, C. Fornaini et al., "Laser-assisted surgery with different wavelengths: a preliminary ex vivo study on thermal increase and histological evaluation," Lasers in Medical Science, vol. 28, no. 2, pp. 497-504, 2013.

[17] P. Vescovi, L. Corcione, M. Meleti et al., "Nd:YAG laser versus traditional scalpel. A preliminary histological analysis of specimens from the human oral mucosa," Lasers in Medical Science, vol. 25, no. 5, pp. 685-691, 2010.

[18] F. Angiero, L. Parma, R. Crippa, and S. Benedicenti, "Diode laser $(808 \mathrm{~nm})$ applied to oral soft tissue lesions: a retrospective study to assess histopathological diagnosis and evaluate physical damage," Lasers in Medical Science, vol. 27, no. 2, pp. 383-388, 2012.

[19] W. Jerjes, T. Upile, Z. Hamdoon et al., " $\mathrm{CO}_{2}$ laser of oral dysplasia: clinicopathological features of recurrence and malignant transformation," Lasers in Medical Science, vol. 27, no. 1, pp. 169179, 2012.

[20] G. Palaia, A. Del Vecchio, A. Impellizzeri et al., "Histological ex vivo evaluation of peri-incisional thermal effect created by a new-generation $\mathrm{CO}_{2}$ superpulsed laser," The Scientific World Journal, vol. 2014, Article ID 345685, 6 pages, 2014. 


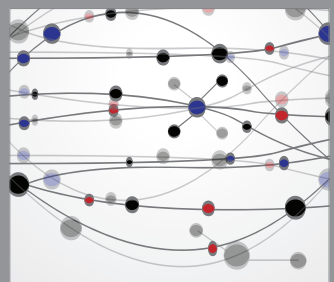

The Scientific World Journal
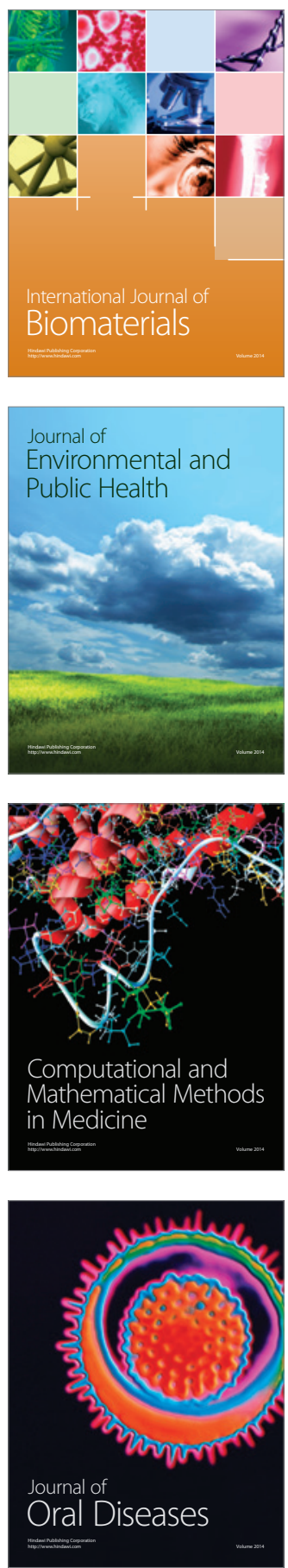
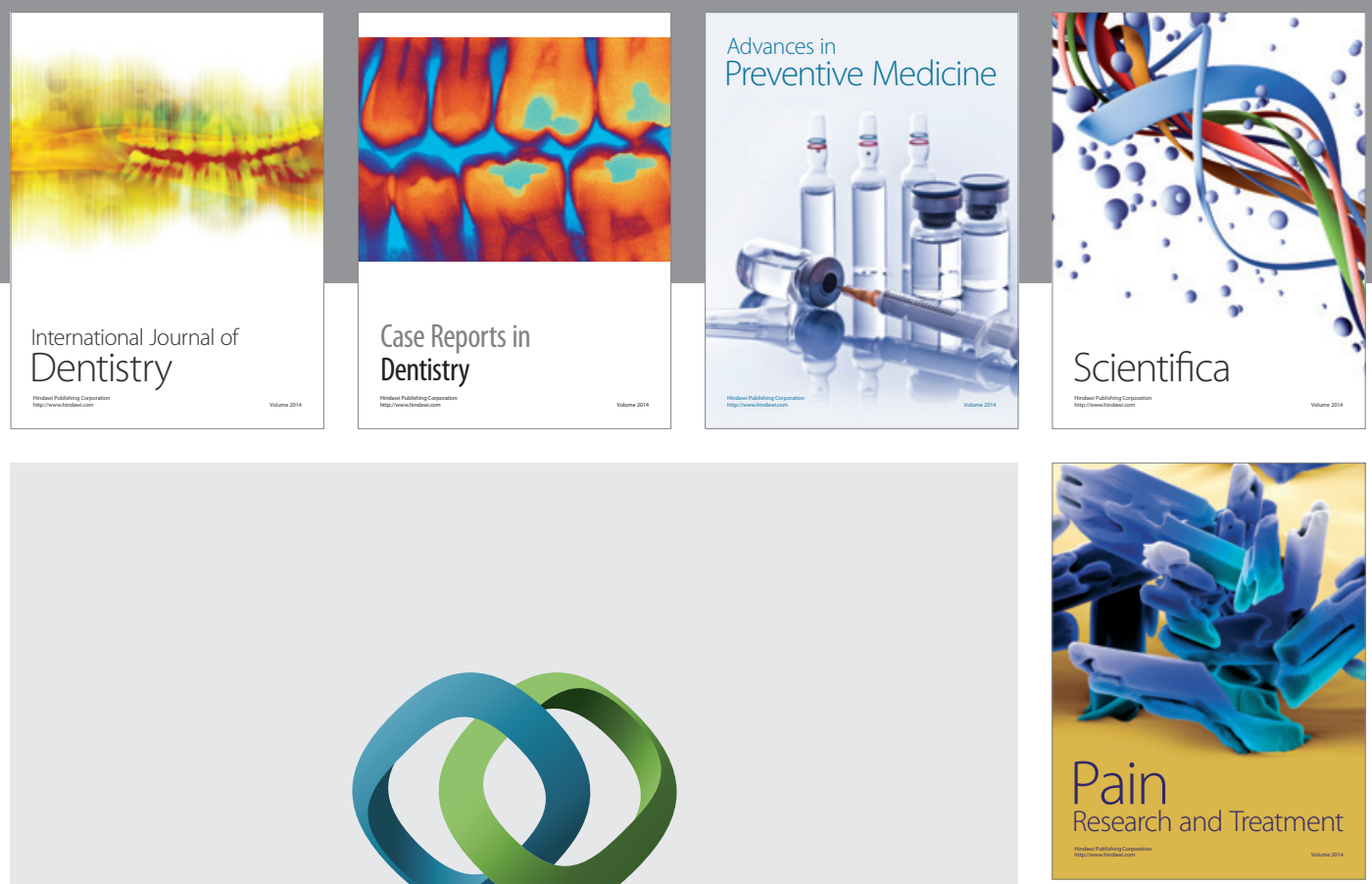

\section{Hindawi}

Submit your manuscripts at

http://www.hindawi.com
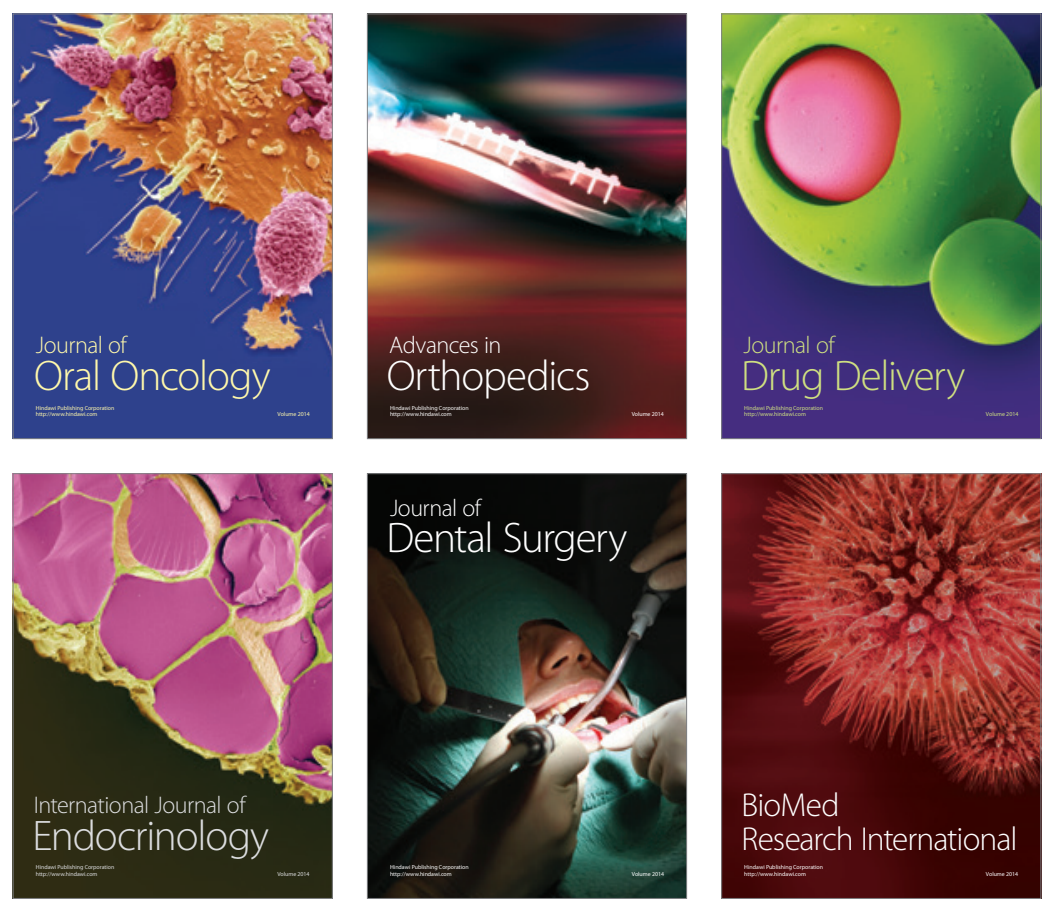

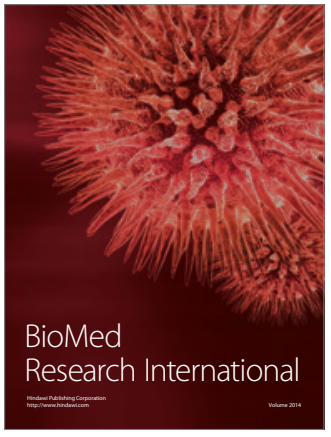

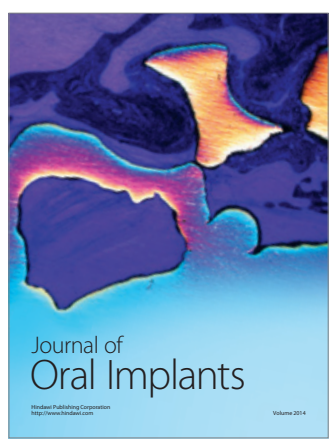
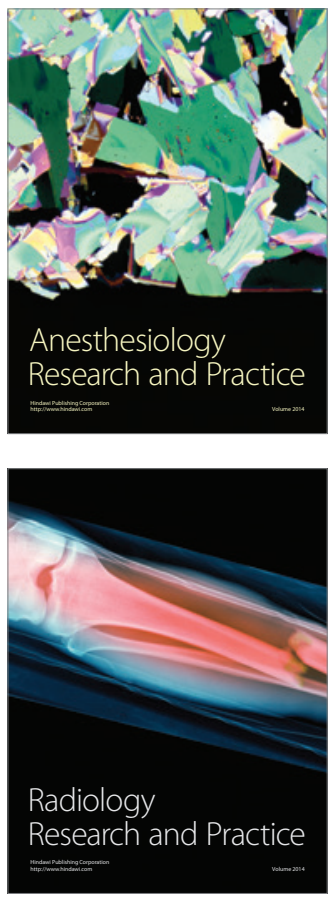